\title{
Experimental Feasibility Study of Estimation of the Normalized Central Blood Pressure Waveform from Radial Photoplethysmogram
}

\author{
Edmond Zahedi ${ }^{1,2^{*}}$, Vahid Sohani ${ }^{1}$, M. A. Mohd. Ali ${ }^{1}$, Kalaivani Chellappan ${ }^{1}$ \\ and Gan Kok Beng ${ }^{1}$ \\ ${ }^{1}$ Department of Electrical, Electronic and Systems Engineering, Faculty of \\ Engineering and Built Environment, Universiti Kebangsaan Malaysia (UKM) \\ ${ }^{2}$ School of Electrical Engineering, Sharif University of Technology, Iran
}

Submitted June 2014. Accepted for publication November 2014.

\begin{abstract}
The feasibility of a novel system to reliably estimate the normalized central blood pressure $\left(\mathrm{CBP}_{\mathrm{N}}\right)$ from the radial photoplethysmogram (PPG) is investigated. Right-wrist radial blood pressure and left-wrist PPG were simultaneously recorded in five different days. An industry-standard applanation tonometer was employed for recording radial blood pressure. The CBP waveform was amplitude-normalized to determine $\mathrm{CBP}_{\mathrm{N}}$. A total of fifteen second-order autoregressive models with exogenous input were investigated using system identification techniques. Among these 15 models, the model producing the lowest coefficient of variation $(\mathrm{CV})$ of the fitness during the five days was selected as the reference model. Results show that the proposed model is able to faithfully reproduce $\mathrm{CBP}_{\mathrm{N}}$ (mean fitness $=85.2 \% \pm 2.5 \%$ ) from the radial PPG for all 15 segments during the five recording days. The low $\mathrm{CV}$ value of $3.35 \%$ suggests a stable model valid for different recording days.
\end{abstract}

Keywords: central blood pressure, radial photoplethsymography, dynamic linear model, system identification

\section{INTRODUCTION}

Cardiovascular diseases (CVD's) continue to remain one of the biggest health issues despite progress in medicine and development of new diagnostic tools. Besides hereditary factors, sedentary life-style and work-related stress contribute to the risk of CVD's in aging populations. Among health conditions, hypertension has probably the most dramatic outlook, especially when occurring with other diseases such as diabetes. The net result is a heavy financial burden, estimated to be USD 2.3 trillion in the USA alone in 2008, with similar trends in other parts of the world [1]. Another unenviable impact is the increase in the dependency ratio, defined as the number of individuals who are retired or too young to work divided by the number of individuals who are in the working age range. This dependency ratio is poised to jump from its current value of

*Corresponding Author: Edmond Zahedi, PhD, Department of Electrical, Electronic and Systems Engineering, Faculty of Engineering and Built Environment, National University of Malaysia, Bangi 43600, Malaysia, Phone: +60-89217099 E-mail: edmond.zahedi@ukm.edu.my. Other authors: v.sohani@eng.ukm.my; mama@eng.ukm.my; gankokbeng@ukm.edu.my; kckalai@ukm.edu.my. 
just above $20 \%$ to more than $35 \%$ in 2030 [1]. In this context, any step towards prevention of CVD's may lighten the amount of healthcare efforts necessary to maintain an adequate quality of life for the population in general and the elderly in particular. As one of the main indicators of cardiovascular health is blood pressure (BP), a myriad of devices are commercially available for the indirect measurement of systolic blood pressure (SBP) and diastolic blood pressure (DBP). Although the two major characteristics of the BP are the peak (SBP) and valley (DBP) leading to their weighted mean arterial pressure $(\mathrm{MAP}=(1 / 3) \times \mathrm{SBP}+(2 / 3) \times \mathrm{DBP})$, the time-varying waveform of the BP $(B P(t))$ has received considerable interest due to the rich amount of cardiovascular-related information [2]. Furthermore, the central $\mathrm{BP}(C B P(t))$ waveform has proven to be very relevant to the diagnostic of CVD [3]. The measurement of $C B P(t)$, done by inserting a catheter through a femoral, carotid or radial artery, is both technically and clinically challenging, especially when long-term measurements are sought [4]. Being invasive, this direct way of blood pressure measurement is strictly limited to the operating theater.

Fortunately, other devices have been made commercially available based on the principle of arterial tonometry and generalized transfer function (GTF) [5]. The principle of operation of these devices is based on a pressure sensitive applanation tonometer which is placed over an artery (generally the radial artery). Upon application of an external pressure equal to the mean arterial pressure, the arterial wall becomes flattened, eliminating radial forces due to the artery wall distension [6]. Under such conditions, the pressure exerted to the tonometer is equal to the blood pressure value in the 'unloaded' artery beneath. In order to get the CBP, an empirically derived GTF is employed [5]. The most established of such devices is the SphygmoCor [7], although other devices using the same principle are also commercially available [8]. Our group investigated the advantages and limitations of these devices. After consideration of all parameters, finally the SphygmoCor was identified as having a stronger validation pool of academic publications [8].

Despite being non-invasive, proper positioning and steadfastly keeping the applanation tonometer over the radial artery proves to be difficult and requires a skilled operator. Another limitation in deployment of continuous blood pressure waveform recording systems using tonometers is their relatively high cost, in the range of dozens of USD. The tonometer is generally made of a very sensitive Mylar pressure transducer, and this expensive transducer is easily prone to malfunction by mishandling especially in developing countries, making the adoption of such devices even more limited in a budget-constrained clinical environment.

To remedy these problems, attempts have been made to non-invasively estimate $B P(t)$ using alternative transduction mechanisms. One such approach is based on the photoplethysmogram (PPG), which is the signal corresponding to the amount of light after it has been attenuated by the vascular bed and surrounding tissues [9]. One of the earliest works in this field has been reported by Millasseau et al. [10] where $B P(t)$ is measured using a finger pressure monitoring device (Finapres) and the PPG is recorded from another finger of the same hand. A limitation of the approach in [10] is the use of the empirical Fourier transform estimate (EFTE), which is known to be sensitive to 
measurement noise [11]. To minimize the effect of noise and error in the EFTE technique, in a previous study, all heart cycles' duration are normalized to exactly one second. As a result, useful information related to the frequency components of $B P(t)$ may have been lost. A more recent work [12] has adopted a similar approach but under a different condition where subjects were asked to do a predefined set of exercises so that their BP varies considerably.

In this work, we propose to investigate the possibility of using the radial PPG to derive $C B P_{N}(t)$, the amplitude-normalized $C B P(t)$, using a linear dynamic model (transfer function). Being a conduit artery, the radial artery offers the advantage of not being affected by any local auto-regulation mechanism. In order to ascertain the reproducibility of our technique, the estimated CBP is evaluated in the course of 5 different days for the same subject. The rest of this paper is organized as follows: Section 1.1 will introduce a brief theoretical background. In Section 2, the data acquisition setup and methods employed to estimate the models are described. Results are presented in Section 3, followed by an interpretation of these results and conclusions in Sections 4 and 5, respectively.

\subsection{Theoretical Background}

\subsubsection{Arterial Diameter Variations with Blood Pressure}

The arterial wall is essentially composed of viscoelastic tissues consisting mainly of elastin and collagen [13], exhibiting a non-linear behavior in response to the arterial pressure $P_{\text {art }}[14]$. Each left ventricle contraction generates a pulse pressure wave which propagates from the aorta and reaches the extremities through the arterial tree. The incidence of the front-end of this pulse pressure causes the arterial diameter to increase and the thickness of the strained arterial wall to decrease accordingly [13]. Eventually, the arterial wall will induce enough force to balance the internal pressure (Figure 1) and a dynamic equilibrium is rapidly reached. A pressure cuff will apply an external pressure $P_{e x t}$, whereas the relationship between the artery diameter $d$ and the transmural pressure $\left(P_{t r}=P_{a r t}-P_{e x t}\right)$ is given in eqn. 1 [14], where $d_{n}$ is the diameter difference from a fully collapsed state to the inflection point, $d_{p}$ is the diameter difference from the inflection point to a fully expanded size, and $C_{\max }$ is the slope of the pressure-diameter curve at the inflection point.

$$
d= \begin{cases}d_{n}+\frac{2 d_{n}}{\pi} \tan ^{-1}\left(\frac{\pi \times C_{\max }}{2 d_{n}} \times P_{t r}\right) & \text { for } P_{\mathrm{tr}}<0 \\ d_{n}+\frac{2 d_{p}}{\pi} \tan ^{-1}\left(\frac{\pi \times C_{\max }}{2 d_{p}} \times P_{t r}\right) & \text { for } P_{\mathrm{tr}} \geq 0\end{cases}
$$

A typical static response of the arterial diameter under the effect of $P_{t r}$ (eqn. 1) is illustrated in Figure 2, with the following physiologically meaningful parameters' values [15]: $C_{\max }=0.15 \mathrm{~mm} /(\mathrm{mmHg}) ; d_{\max }=3.22 \mathrm{~mm} ; d_{n}=2.00 \mathrm{~mm} ; d_{p}=d_{\max }-d_{n}=$ $1.22 \mathrm{~mm}$. When $P_{e x t}=P_{\text {mean }}$, i.e. $P_{t r}=0$, the artery is at its maximum compliance (change in vessel volume per unit change of blood pressure). 


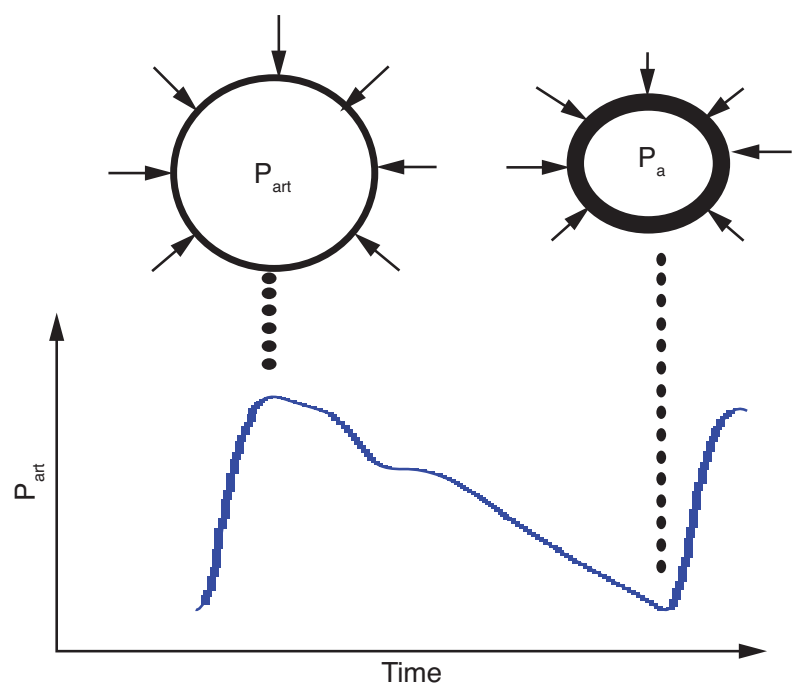

Figure 1. Conceptual cross-section of the arterial wall subjected to the arterial pressure $\left(\mathrm{P}_{\mathrm{art}}\right)$ counter-balanced by the pressure emanating from the distension of the arterial wall (arrows). Large diameter artery with thin wall at systole (left). Small diameter artery with thick wall at diastole (right).
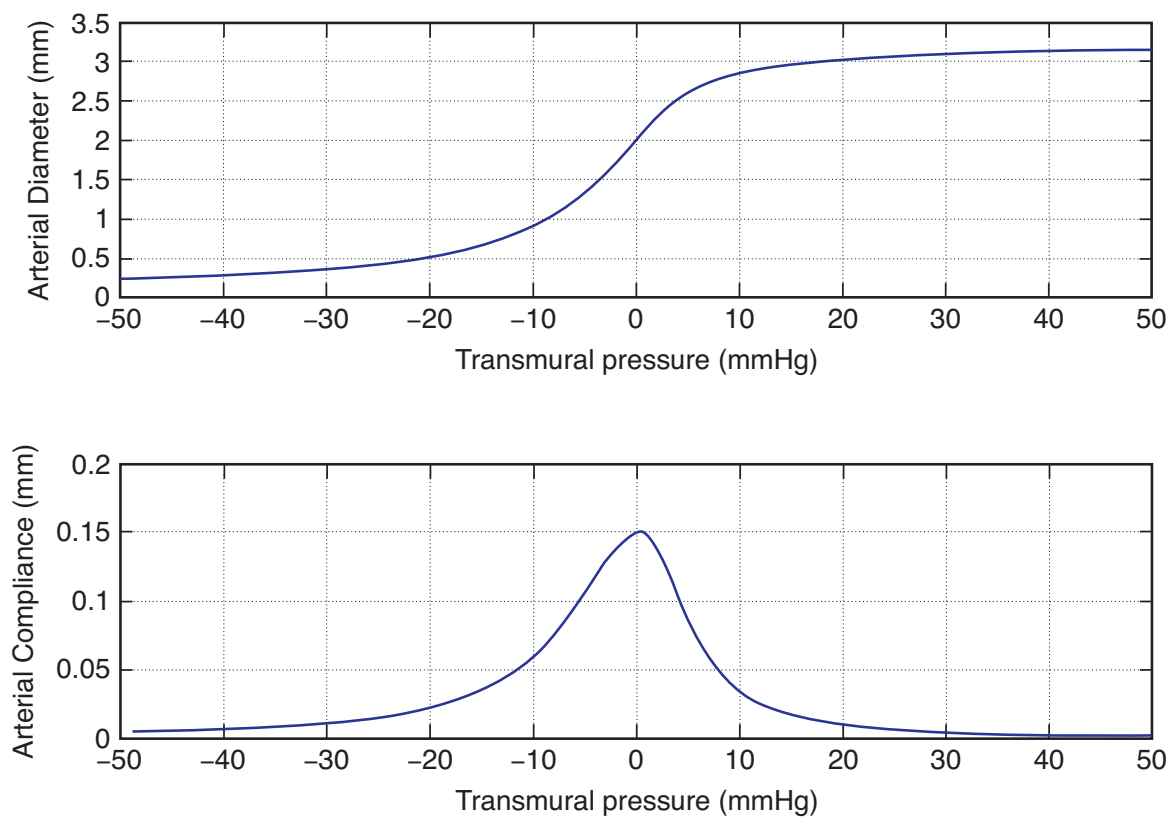

Figure 2. Typical behavior of the arterial diameter (top) and compliance (bottom) versus transmural pressure $P_{t r}$. See text for parameter values. 
It is clear that the dynamic response of the artery diameter depends on the operating point set by the transmural pressure $\left(P_{t r}\right)$. Setting $P_{e x t}$ equal to the mean arterial pressure will place the operating point at the maximum arterial wall compliance. Under such a condition, $P_{t r}$ will oscillate around zero and maximum amplitude oscillations are obtained. During applanation tonometry, the operator strives to reach this point by adjusting the exerted force on the probe so that amplitude variations become maximal, an indication that $P_{\text {ext }}=P_{\text {art }}$.

\subsubsection{Genesis of the Photoplethysmogram}

When a beam of monochromatic light of intensity $I_{0}$ at wave length $\lambda$ passes through a distance $d$ of tissues consisting of muscle, fat, venous and arterial blood, and if reflection and scattering can be neglected, the resulting amount of light intensity known as $P P G(t)$ can be roughly approximated by the Beer-Lambert's law (2):

$$
P P G(t)=I_{0} \times e^{-\alpha_{m} d_{m}(t)} \times e^{-\alpha_{f} d_{f}(t)} \times e^{-\alpha_{v} d_{v}(t)} \times e^{-\alpha_{a} d_{a}(t)}
$$

where $\left\{\alpha_{m}, \alpha_{f}, \alpha_{v}, \alpha_{a}\right\}$ are the wavelength-dependent optical extinction coefficients, and $\left\{d_{m}(t), d_{f}(t), d_{v}(t), d_{a}(t)\right\}$ are the time $(t)$ varying path lengths for muscle, fat, venous and arterial blood, respectively. For the rest of this paper, we will refer to $P P G(t)$ as PPG.

As biological tissues are far from being homogenous, in reality, the light beam will also undergo reflections caused by various layers of tissue, arterial and venous blood, bone and arterial wall(s) [9]. The net result of this combination is a pulsatile (alternate) component (AC) superposed to a constant amplitude intensity (DC). The source of the AC signal is the arterial blood; therefore, it is synchronized with the heart pumping cycle. Under normal perfusion circumstances, the peak-to-peak amplitude of the AC component of the finger PPG is approximately $10 \%$ of the DC. At the systolic instant, the arterial diameter is maximum (Figure 1); therefore, the amount of collected light after the light beam has traveled through the tissue layers is minimum (eqn. 2). Similarly, the amount of collected light will be at its maximum at the diastolic instant. It is common practice to negate the AC part of the PPG so that the systolic instant corresponds to a peak and the diastolic to a valley (Figure 3).

\subsubsection{Linear, Dynamic Relationship Approximation}

A simple consideration of eqns. 1 and 2 indicates clearly that the relationship between $B P(t)$ and PPG is highly non-linear. However, when operation around $P_{\mathrm{tr}}=0$ is considered, a linear approximation may be assumed. Therefore, it may be plausible to propose a linear, dynamic (due to arterial elasticity) relationship between $B P(t)$ and PPG as long as the pulse pressure (SBP - DBP) does remain within the linear range of variation of the arterial diameter $d$ versus $P_{\mathrm{tr}}$. For example, under the conditions for the graph in Figure 2 and an operating point defined by $P_{\mathrm{tr}}=0$, a pulse-pressure of less than $15 \mathrm{mmHg}$ would satisfy this condition. In practice, however, the pulse pressure is generally more than $15 \mathrm{mmHg}$ and such a deviation from the $15 \mathrm{mmHg}$ zone will reduce the fitness of the model output. 

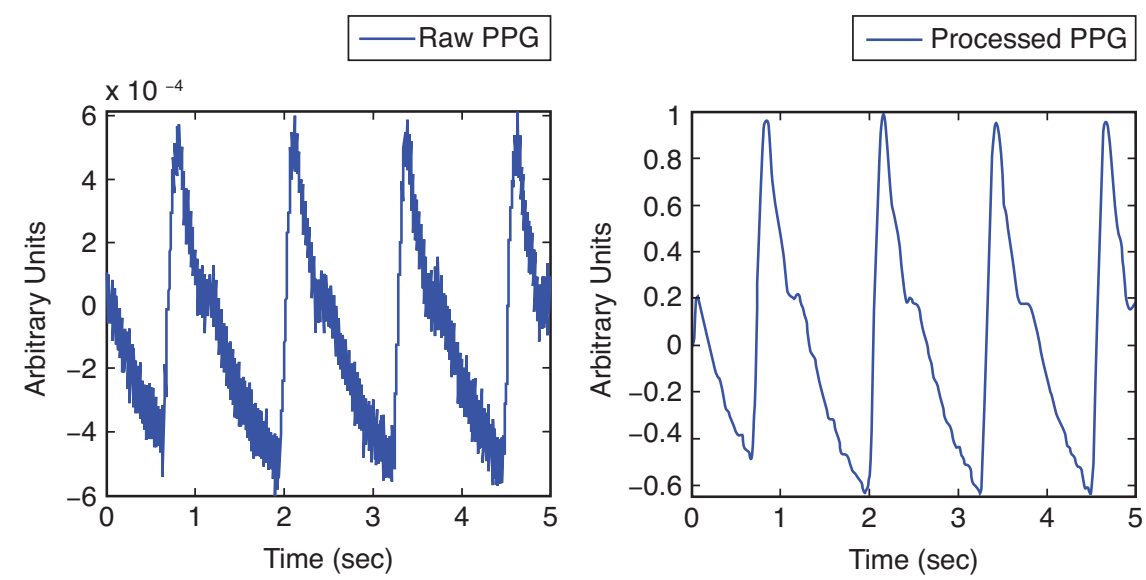

Figure 3. Sample of a radial PPG from sensor output. Left panel: raw data. Right panel: after low-pass filtering $(15 \mathrm{~Hz})$, detrending and peak amplitude normalization to unity by dividing the signal by its maximum value.

The main hypothesis of our work is the existence of a linear, dynamic relationship between the normalized-amplitude radial $\mathrm{CBP}\left(C B P_{N}\right)$ and the radial PPG. The verification of the existence of such a relationship will therefore be our objective in this paper. Furthermore, we will investigate the stability of such a relationship over different days for the same subject.

\section{METHODS}

\subsection{Data Acquisition Protocol}

This study conforms to the Declaration of Helsinki [16] was approved by the ethical committee of the university hospital (Hospital Universiti Kebangsaan Malaysia) with reference number: UKM 1.5.3.5/244/FF-2013-409. Data were acquired from a healthy, non-smoking male (25 years) who did not have any cardiovascular risk factor. The subject was briefed by introducing him to the setup one week prior to the experiment.

The subject gave informed consent, fasted overnight and refrained from any heavy physical activity. The subject was placed in a temperature controlled, noise-free room with constant illumination for 15 minutes. Then he was made to lie in the supine position on a low-rise bed for two minutes. To avoid any motion artifact, the subject was instructed to refrain from talking or moving during data acquisition. The subject was called for a total of five different days spread over 2 weeks. On each day, the same protocol (as described above) was strictly followed, and a set consisting of five records were obtained. The setup was operated by a person trained by the manufacturer (AtCor Medical, Australia).

\subsection{Recording Setup}

Figure 4 shows the setup where a reflective-type PPG sensor (g.PULSEsensor, g.Tec Medical Engineering, Austria) was attached to the left wrist of the subject. The output 


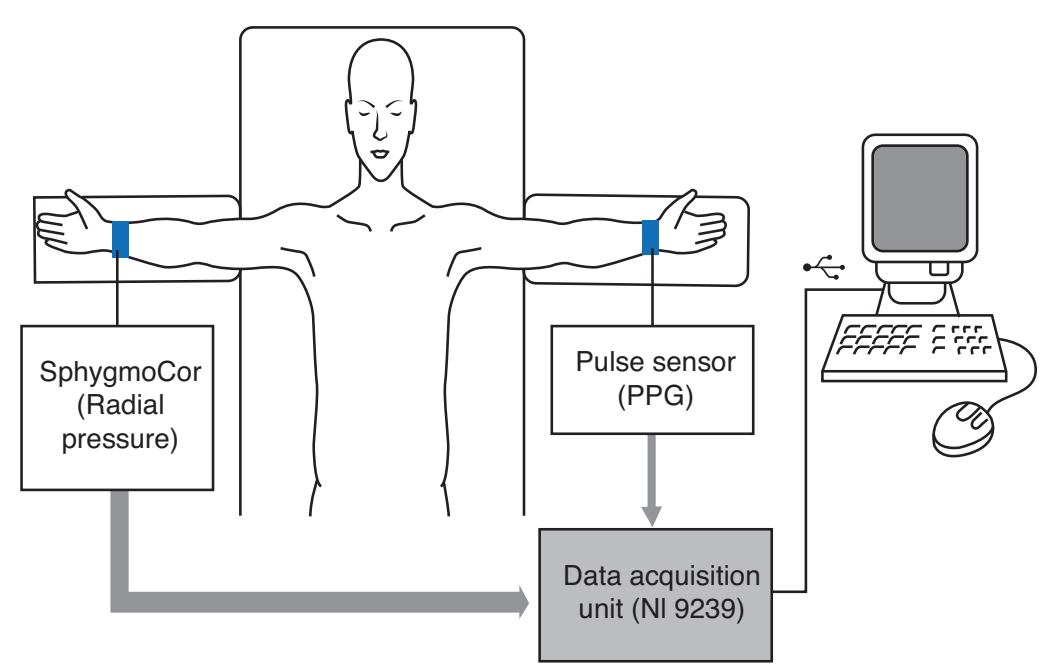

Figure 4. Schematic of the data acquisition setup.

of this sensor and associated amplifier circuit is the AC component of the PPG. A tonometer (AtCor Medical, Australia) was attached to the right wrist. Two arm rests were specially fabricated to reach the bed's height where the subject laid on the supine position. This ensured that the subject's arms remained comfortably extended during data acquisition. The wrist tonometer output was connected to a SphygmoCor unit (AtCor Medical, Australia), measuring the radial BP. The CBP is internally computed by the SphygmoCor using the manufacturer's proprietary algorithm; therefore, it is a derived CBP. Care was taken to attach each sensor as close to the bone prominence as possible over the respective left and right radial arteries. Both PPG and tonometer positions were manually adjusted with great care so as to receive the maximum amplitude signal, resulting in the best quality in terms of signal-tonoise ratio. The SphygmoCor device has two analog outputs: the measured radial pressure and reconstructed CBP. These signals as well as the wrist PPG sensor output were digitized by a 24-bit analog-to-digital data acquisition module (NI 9239, National Instruments, USA). A LabView program (National Instruments, USA) was utilized to record $180 \mathrm{~s}$ (three minutes) of data at a sampling rate of $1613 \mathrm{~Hz}$ (default setting). All data were saved under ASCII format on a personal computer hard disk for off-line processing.

\subsection{Signal Processing}

In order to prepare the data for identifying the model structure and parameters, the following steps were implemented using MATLAB (The Mathworks, Inc., Massachusetts, USA) according to the standard guidelines [11]. 


\subsubsection{Low-Pass Filtering}

Using an in-house developed code, a linear phase finite impulse response (FIR) lowpass filter (LPF) was implemented with a cut-off frequency of $15 \mathrm{~Hz}$. The choice of the cut-off frequency is based on the useful frequency components of the CBP and PPG [9]. All recorded signals were low-pass filtered using the same LPF. The code of this LPF is presented in Appendix A.

\subsubsection{Time Alignment}

To be able to record the signals of interest on a long term basis, the SphygmoCor device was put in the pulse wave analysis (PWA) mode. According to the manufacturer, the reconstructed CBP output signal available from the SphygmoCor device has been aligned in time, making the absolute value of time arbitrary. Therefore, we performed a time alignment of the PPG with the CBP. This approach allowed us to simplify delay estimation during model identification by simply setting the value of the delay parameter $n_{k}$ to zero. Details of the model identification procedures are explained in section 2.4 .

\subsubsection{Resampling}

The high sampling rate of $1613 \mathrm{~Hz}$ clearly oversamples the data as the useful signal frequency is only up to $15 \mathrm{~Hz}$. Oversampling produces an unnecessarily large size of the data records, requires more computation time, and results in the compaction of the poles and zeros [11]. To avoid these problems, all signals were resampled at $100 \mathrm{~Hz}$.

\subsubsection{Data Segment Selection}

For model identification purposes, two types of data are required: estimation and validation [11]. In our study, these input data were selected through careful visual inspection, the criteria being to avoid any occurrence of motion artifact or extraneous measurement noise. For each recording session, three non-overlapping segments were selected. The duration of all selected segments (estimation and validation) was five seconds, corresponding approximately to five heartbeats. Given that the signals were recorded during five different days, the above segment selection exercise resulted in a total of 15 (3 segments $\times 5$ days) data segments. We observed that there was no significant difference in the model parameters if a much larger segment was selected, as long as the mentioned segment remained noise-free. The major advantage of choosing smaller segments is shorter computation time and more uniform (5-sec segments) approach towards all data records.

\subsubsection{Detrending Procedure}

As any existing trend in the signal will affect the performance of the system identification procedure [11], the linear trend was independently eliminated from each of the data segments.

\subsubsection{Amplitude Normalization}

As a final preprocessing step, the recorded data sample values of the signals were divided by their own segment maximum. This ensured that for both CBP and PPG, the 


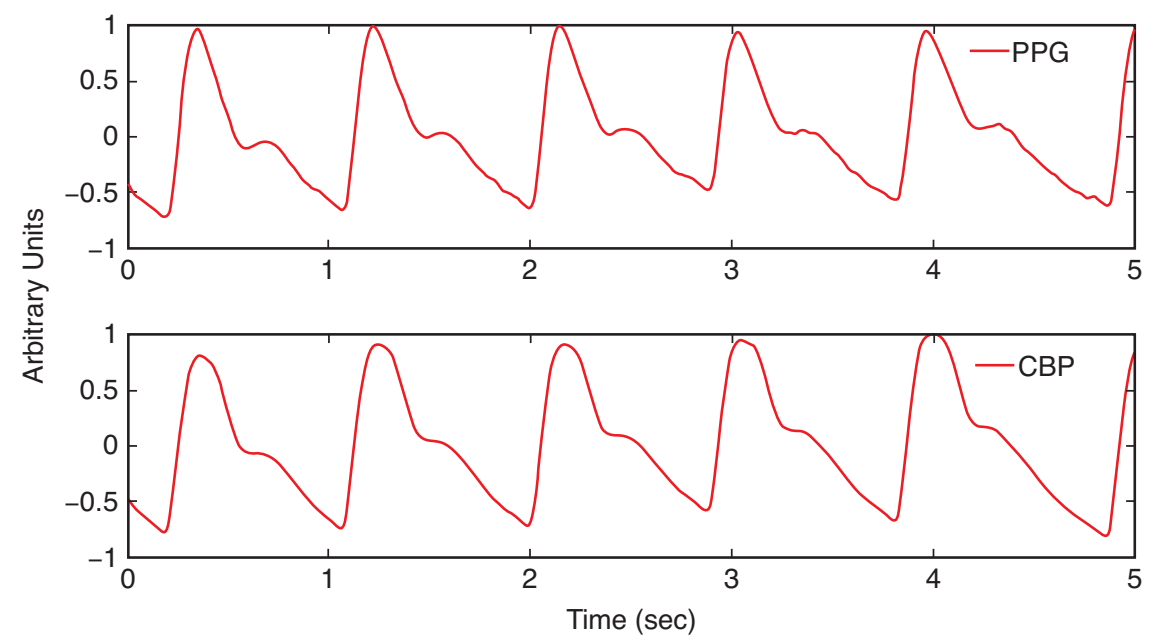

Figure 5. Typical measured PPG (top panel) and $\mathrm{CBP}_{\mathrm{N}}$ (lower panel) traces after preprocessing.

peak value during each segment was one. The most negative value of each of the signals depends on the particular waveform. Figure 5 shows a typical 5-second segment of the PPG and $C B P_{\mathrm{N}}$ after preprocessing.

\subsection{Model Determination}

The procedure for signal processing is shown in Figure 6. The preprocessing steps have been explained in Section 2.3 and the remaining parts will be discussed in detail here. The System Identification Toolbox in MATLAB was utilized to perform the necessary processing in this section. Our aim is to determine the best linear, parametric model (Figure 7) giving the closest output $y(t)$ to the observed signal defined by:

$$
y(t)=\sum_{k=1}^{\infty} h(k) x(t-k)+\varepsilon(t)
$$

where $t$ is the discrete time, $h(k)$ is the impulse response of the model, $x(t)$ is the input and $\varepsilon(t)$ is an additive noise term which reflects the inaccuracies of the model.

\subsubsection{Model Structure}

The first step in the model determination is to find the adequate model structure. Considering a linear, parametric relationship between the input $\left(C B P_{N}\right)$ and the output (PPG), the auto-regressive with exogenous (ARX) input was naturally our first choice. Although the auto-regressive moving-average with exogenous (ARMAX) input model was also investigated, our preliminary tests showed that ARX outperformed ARMAX 


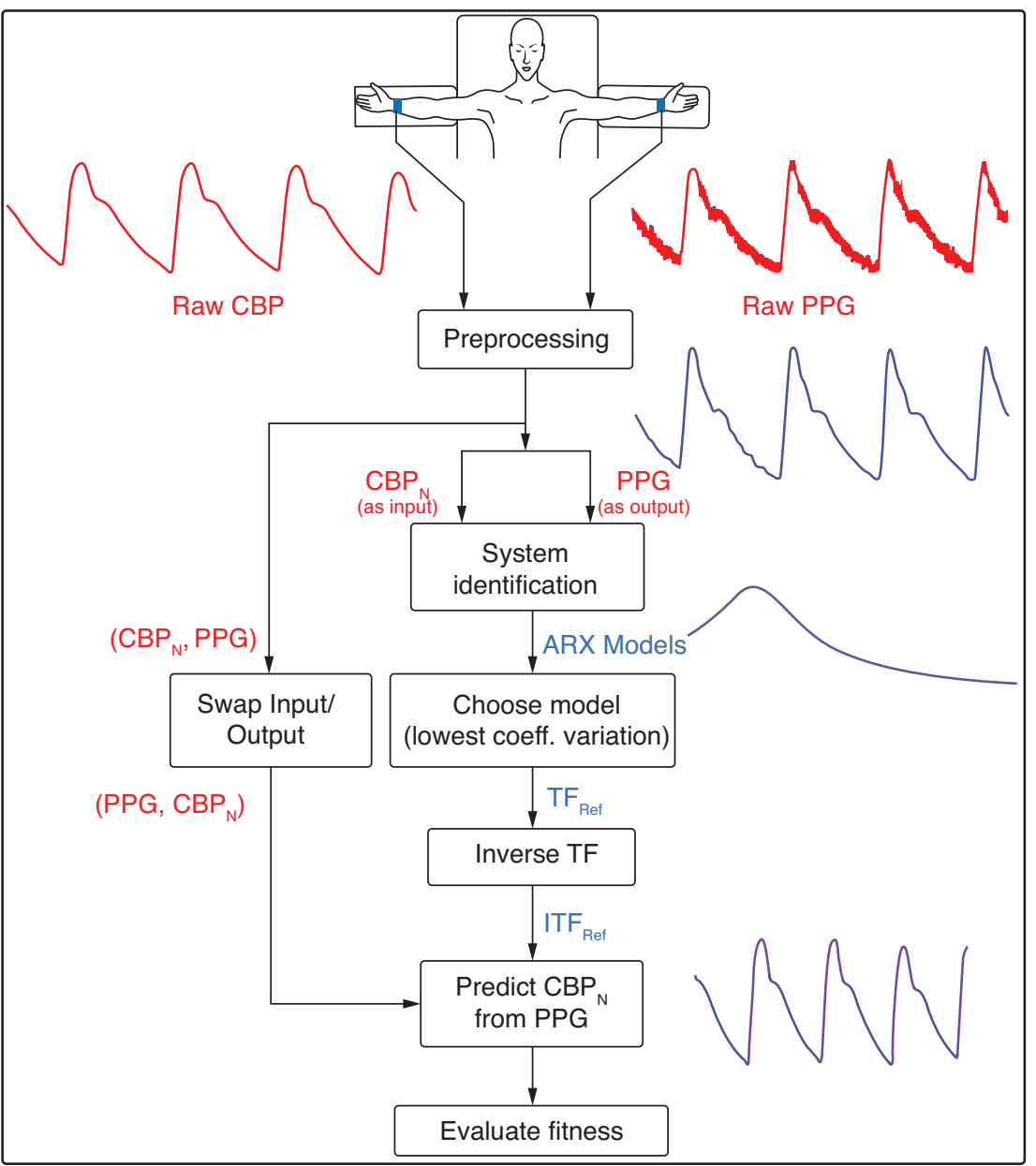

Figure 6. Steps involved in the course of the model identification and evaluation.

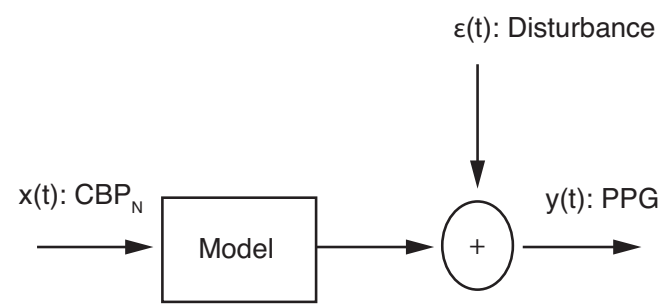

Figure 7. General representation of the modeling approach. The normalized central blood pressure $\left(C B P_{N}\right)$ is the input and radial photoplethysmogram (PPG) the output. 
in terms of goodness of fit and residual noise density. Furthermore, the ARX model is known to have good numerical stability properties and less bias during estimation of the model's parameters [11]. In the ARX model, the input $x(t)$ and output $y(t)$ at the discrete sample time $t$ are related by the following equation:

$$
y(t)=\sum_{k=0}^{n_{d}} a(k) \times x\left(t-k-n_{k}\right)+\sum_{k=1}^{n_{b}} b(k) \times y(t-k)+\varepsilon(t)
$$

where $n_{a}$ is the order of the denominator (number of poles) in the ARX transfer function, $n_{b}$ is the order of the numerator (number of zeros plus one), $n_{k}$ is the inputoutput delay, $a(k)$ and $b(k)$ are the numerator and denominator coefficients of the transfer function, respectively, and $\varepsilon(t)$ is the residual noise. By convention, we will refer to the above model as ARX $\left[n_{a} n_{b} n_{k}\right]$.

\subsubsection{Model Order}

Orders from 1 to 4 have been investigated using the following goodness of fit indices:

a) Fitness value

The Fitness value is defined as [11]:

$$
\text { Fitness }=100 \times\left(1-\frac{\sqrt{\sum_{t=1}^{N}(y(t)-\hat{y}(t))^{2}}}{\sqrt{\sum_{t=1}^{N}(y(t)-\bar{y})^{2}}}\right)
$$

where

$y(t)$ is the measured signal at instant $t$,

$\hat{y}(t)$ is the estimated model output at instant $\mathrm{t}$,

$\bar{y}$ is the mean of the measured signal, and

$N$ is the number of data samples.

b) Final prediction error (FPE)

An alternative way to assess the goodness of fit is to use the Akaike's Final Prediction Error (FPE) [11] given by:

$$
\mathrm{FPE}=V \times\left(\frac{1+\frac{p}{N}}{1-\frac{p}{N}}\right)
$$

where $p$ is the number of estimated parameters and the loss function $V$ is defined as:

$$
V=\operatorname{det}\left(\frac{1}{N} \sum_{k=1}^{N} \varepsilon\left(k, \theta_{N}\right)\left(\varepsilon\left(k, \theta_{N}\right)\right)^{T}\right)
$$



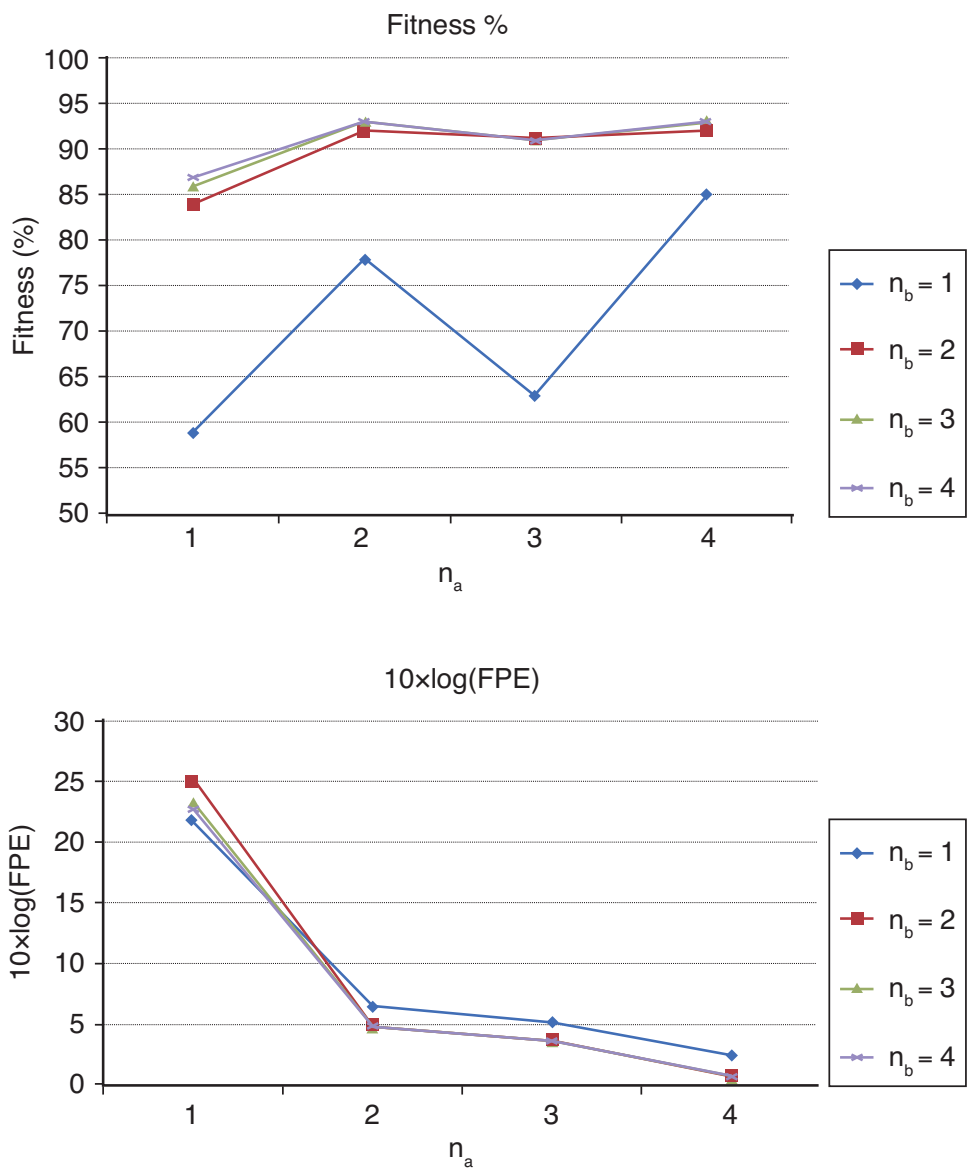

Figure 8. Effect of model order with typical goodness of fit defined by Fitness and FPE values.

where $\theta_{\mathrm{N}}$ represents the vector of estimated parameters from the $N$ data samples, $\varepsilon$ is the residual error and $\operatorname{det}()$ is the matrix determinant operator [7]. In brief, eqn. 6 shows that as long as the number of the model's parameters is very small compared to the number of samples $(p / N<<1)$, the FPE is close to the loss function. Adding more parameters will penalize the FPE so that different model orders can be fairly compared, avoiding a model with numerous parameters to apparently outperform another model with a lesser number of parameters.

Figure 8 shows typical Fitness and FPE values obtained for various ARX $\left[\mathrm{n}_{\mathrm{a}} \mathrm{n}_{\mathrm{b}} \mathrm{n}_{\mathrm{k}}\right]$ model orders ranging from ARX [ $\left.\begin{array}{lll}1 & 1 & 0\end{array}\right]$ to ARX [ $\left.\begin{array}{lll}4 & 4 & 0\end{array}\right]$. In all models, the delay has been fixed to zero (refer to Section 2.3.2); therefore, $n_{k}=0$. For $n_{b} \geq 2$, the values of the Fitness (eqn. 5) become similar as soon as $n_{a} \geq 2$ (Figure 8, top panel). It can also be seen (Figure 8 , bottom panel) that for values of $n_{b} \geq 2$, increasing $n_{a}$ above 2 does not significantly impact the FPE. 


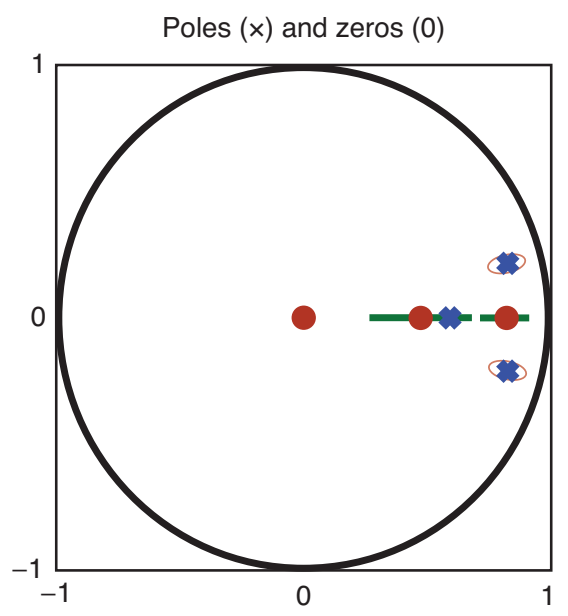

Figure 9. Poles and zeroes of a typical [PPG $\left.\rightarrow C B P_{N}\right]$ model (ARX [3 300$]$ structure). The contours (horizontal on the real axis) represent the $95 \%$ confidence interval. The pole-zero cancellation is caused by order overestimation. Blue (x): poles, Red (o): zeros.

As choosing a high order for a particular model would only make the model better fit the noise instead of the signal of interest [11], the 95\% confidence interval of the poles-zero plots was investigated for orders above two (e.g., ARX [ [ $\left.\begin{array}{lll}3 & 3 & 0\end{array}\right]$ ). See Appendix B for details of computation of the confidence interval.

It was observed that for higher orders, a clear pole-zero cancellation occurred irrespective of the selected data segment (Figure 9). Therefore, the ARX [2 20 ) model was selected as having the optimum numbers of poles and zeros.

\subsubsection{Goodness of Fit Matrix}

Each one of the $15 C B P_{\mathrm{N}} / \mathrm{PPG}$ data segments was utilized to estimate the parameters of

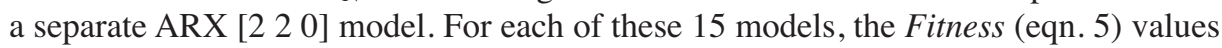
were computed on all 15 data segments, resulting in a $15 \times 15$ goodness of fit matrix. Each row in this Fitness matrix corresponds to a segment and each column to a different ARX [l 220$]$ model.

\subsubsection{Reproducibility of the Models}

Using the goodness of fit matrix as defined in the previous section, the average, median and coefficient of variation $(\mathrm{CV})$ of the Fitness were computed on each column for each of the 15 models:

$$
C V=\frac{\sigma}{\mu}
$$

where $\sigma$ and $\mu$ are the standard deviation and mean of the Fitness values for a particular model, respectively. 


\subsection{5. $C B P_{N}$ Estimation from Radial $P P G$}

Once model parameters were determined using the 15 data segments, the model giving the lowest $C V$ value was selected as the reference model $\left(T F_{\text {Ref }}\right)$. This choice has been preferred over selecting the model with the highest average Fitness. Indeed, our preliminary results indicated that the values of the Fitness obtained for all 15 models were close; thus we gave more weight to the reproducibility of the models rather than the absolute value of their Fitness in the selection of $T F_{\text {Ref. }}$ As all zeros of $T F_{\text {Ref }}$ were located within the unit circle, the inverse of the $T F_{\text {Ref }}\left(I T F_{\text {Ref }}\right)$ was easily obtained by interchanging the numerator with the denominator of the transfer function. Finally, by using $I T F_{\text {Ref }}, C B P_{\mathrm{N}}$ was estimated from the radial PPG for all 15 segments.

\section{RESULTS}

\subsection{Model Determination for a Single Day: $\left[\mathrm{CBP}_{\mathbf{N}} \rightarrow\right.$ PPG]}

In order to ascertain the validity of our approach, an ARX [ 2220$]$ model was obtained for the records obtained on the first day. The estimation segment was used to determine the model parameters. The input of this model consisted of the $\mathrm{CBP}_{\mathrm{N}}$ and the output was the PPG. Figure 10 shows the predicted PPG and the measured PPG for the two validation data segments. The respective values for Fitness are $88 \%$ and $90 \%$ (mean of $89 \%$ ). The frequency response and $95 \%$ confidence interval for this particular model are shown in Figure 11.
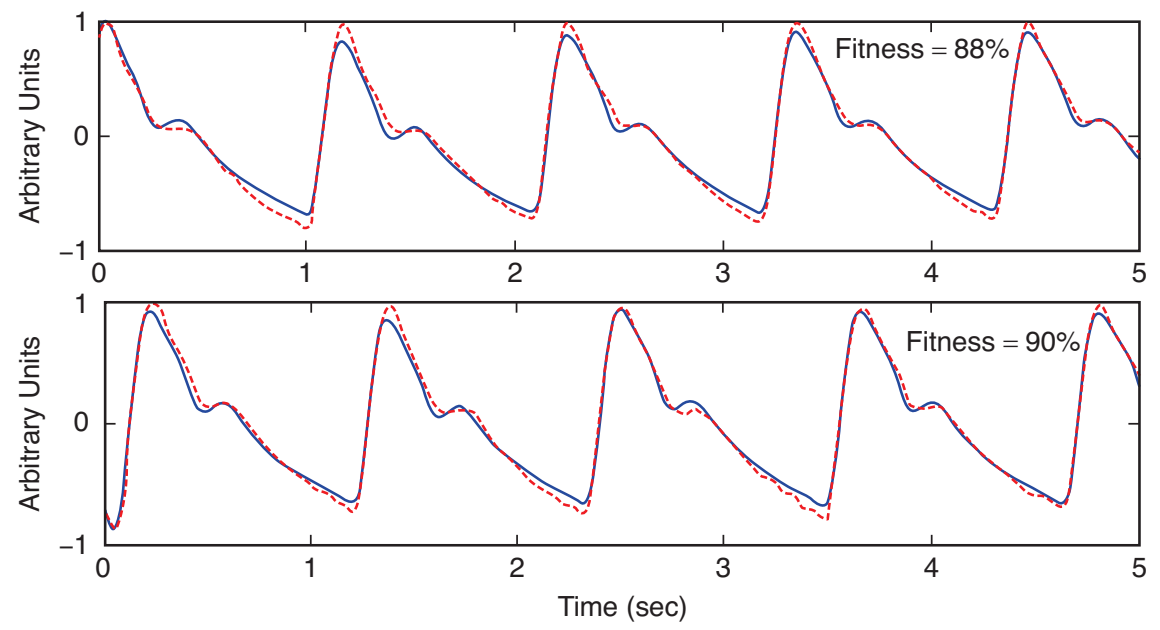

Figure 10. Output of the $\left[C B P_{N} \rightarrow\right.$ PPG] model: predicted PPG (dotted red) computed using an ARX [l 220 ] model and $\mathrm{CBP}_{\mathrm{N}}$ as input and recorded PPG (solid blue). Top panel: validation segment 1. Bottom panel: validation segment 2 . 

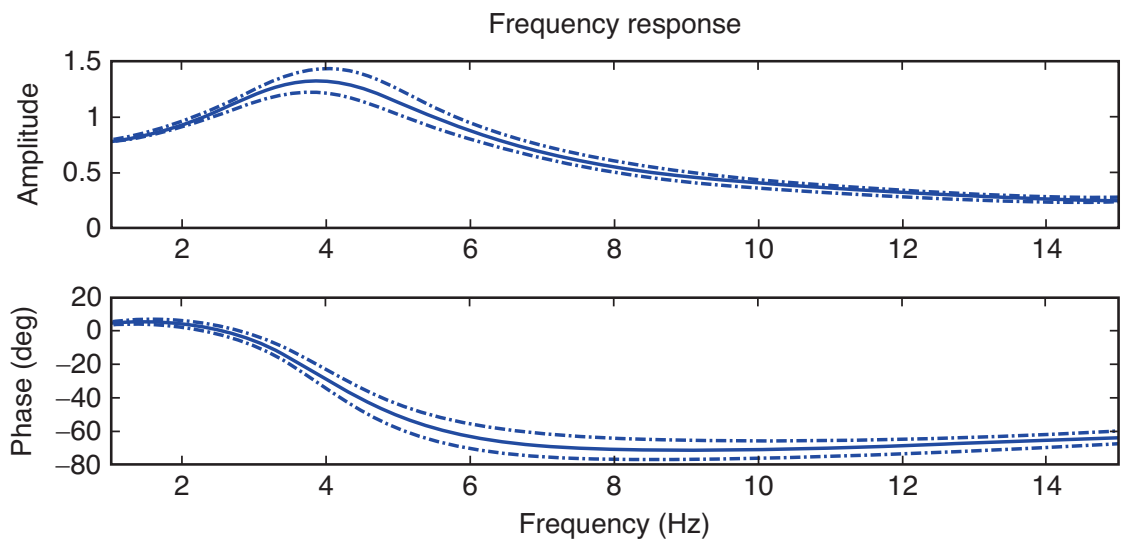

Figure 11. Single [CBP $\rightarrow$ PPG] model: magnitude (top) and phase (bottom) of the frequency response of the same ARX [ $\left.\begin{array}{lll}2 & 2 & 0\end{array}\right]$ model for the data Figure 10. The dotted lines represent the $95 \%$ confidence interval of the frequency response.
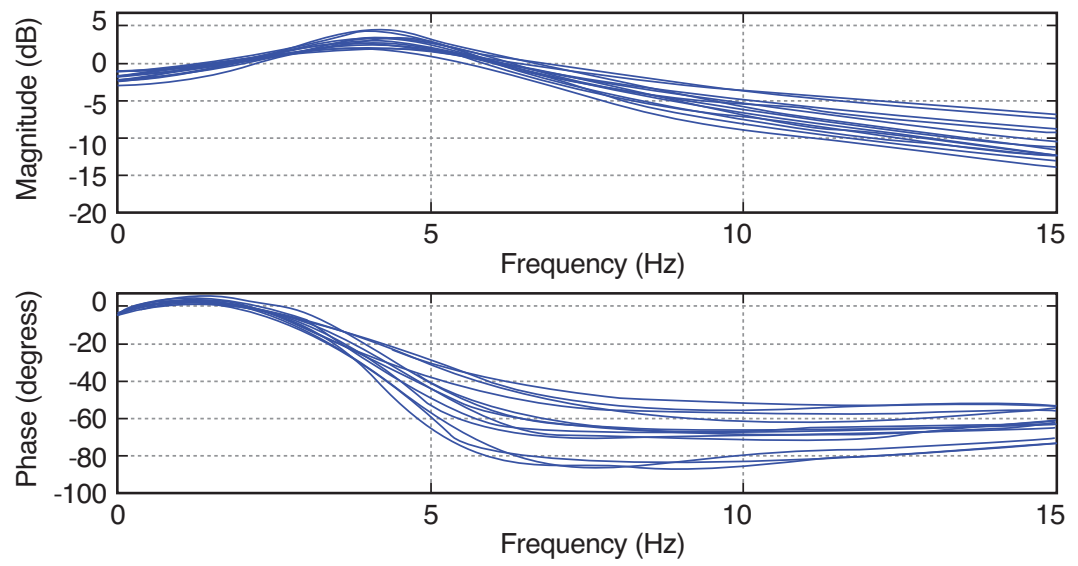

Figure 12. Frequency responses of fifteen $\left[C B P_{N} \rightarrow\right.$ PPG] models: magnitude (top) and phase (bottom) of the frequency response of the fifteen ARX [ $\left.\begin{array}{lll}2 & 2 & 0\end{array}\right]$ models.

\subsection{1. $\left[C B P_{N} \rightarrow P P G\right]$ Models Obtained for all Five Days}

The procedure explained in Section 3.1 was implemented and the parameters of the 15 models were obtained ( 3 segments for each of the 5 days). Figure 12 shows the magnitude and phase of the frequency response of these 15 models. 


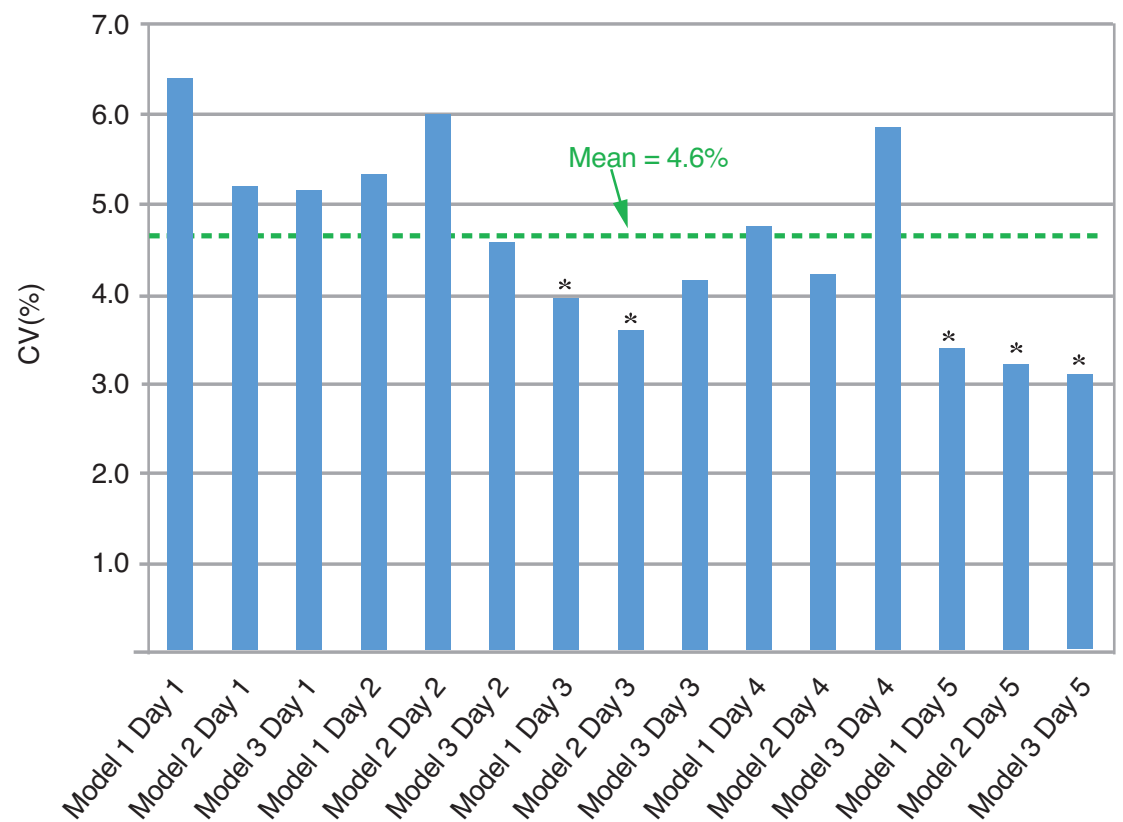

Figure 13. $\left[C B P_{N} \rightarrow\right.$ PPG] models: coefficient of variation $(\mathrm{CV})$ of the Fitness values computed over 5 different days. ${ }^{*} C V \leq 4 \%$.

\subsubsection{Determination of the Reference $\left[C B P_{N} \rightarrow P P G\right]$ Model}

Results shown in Figure 12 suggests a relatively reproducible model as the magnitudes and phases of all 15 models estimated over five days seem consistent. However, the question of variability of the extracted model can only be answered when the Fitness value of each particular model is obtained for all segments recorded throughout the five days.

The $C V$ of the Fitness value for all 15 models are exhibited in Figure 13. Models estimated using the $7^{\text {th }}, 8^{\text {th }}, 13^{\text {th }}, 14^{\text {th }}$ and $15^{\text {th }}$ segments (marked by “*” in Figure 13) have all their $C V$ 's less than or equal to $4 \%$. The lowest $C V(3.1 \%)$ was observed for the last model (Model 3 Day 5) with a Fitness value of $84.7 \%$. Therefore, this particular model was selected as our reference model $\left(T F_{R e f}\right)$. The Fitness of $T F_{R e f}(84.7 \%)$ is very close to the mean value of the Fitness for all 15 models $(84.8 \%)$. The values of the parameters for $T F_{\text {Ref }}$ are given in Table 1.

\subsection{3. $\left[P P G \rightarrow C B P_{N}\right]$ Model}

Using the reference model $T F_{R e f}$ as determined in the previous section, the inverse transfer function $I T F_{R e f}$ was obtained by exchanging the numerator and the denominator. The pole-zero plot of this reference model (Figure 14) shows that the poles are within the unit circle ensuring a stable model. A sample of the reconstructed $C B P_{N}$ when the radial PPG is the input is shown in Figure 15. The values of the Fitness 
Table 1. Coefficients of the selected reference model $T F_{R e f}$ among all 15 models. $T F_{R e f}$ corresponds to the last model (Model 3 Day 5) in Figure 13.

\begin{tabular}{lcccc}
\hline Model & \multicolumn{3}{c}{$\left[\mathbf{a}_{\mathbf{0}} \mathbf{a}_{\mathbf{1}} \mathbf{a}_{\mathbf{2}}\right]$} & \multicolumn{2}{c}{$\left[\mathbf{b}_{\mathbf{0}} \mathbf{b}_{\mathbf{1}}\right]$} \\
\hline ARX[2 2 2 0] & {$\left[\begin{array}{llll}1 & -1.597 & 0.6702\end{array}\right]$} & {$\left[\begin{array}{llll}0.3571 & 0.2931\end{array}\right]$} \\
\hline
\end{tabular}

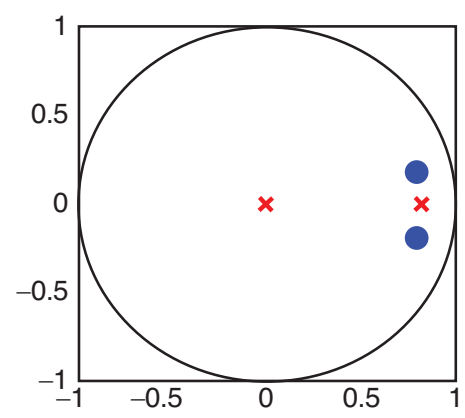

Figure 14. Locus of the poles $(\times)$ and zeros $(\bullet)$ of the inverse reference model $I T F_{R e f}$ for $\left[\mathrm{PPG} \rightarrow \mathrm{CBP}_{\mathrm{N}}\right]$ model

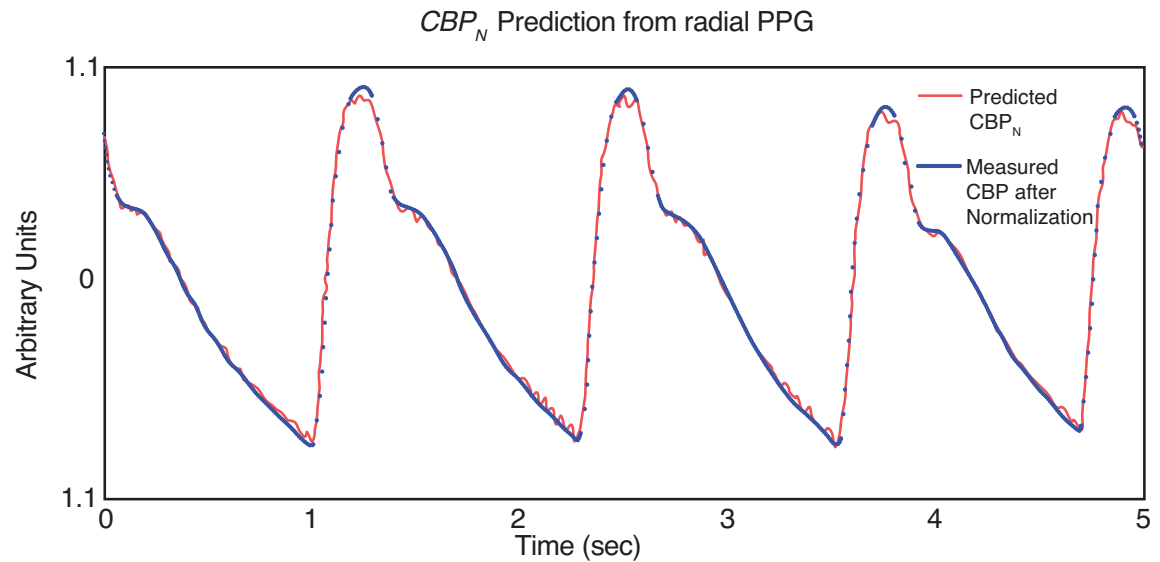

Figure 15. Output of the $\left[\mathrm{PPG} \rightarrow C B P_{N}\right]$ model. Solid red: typical predicted $C B P_{N}$ using $I T F_{R e f}$ and radial PPG as input. Dotted blue: $C B P_{N}$ obtained through amplitude-normalization of the SphygmoCor output. Note that the considered data segment is different from the one on which the reference model has been obtained. 


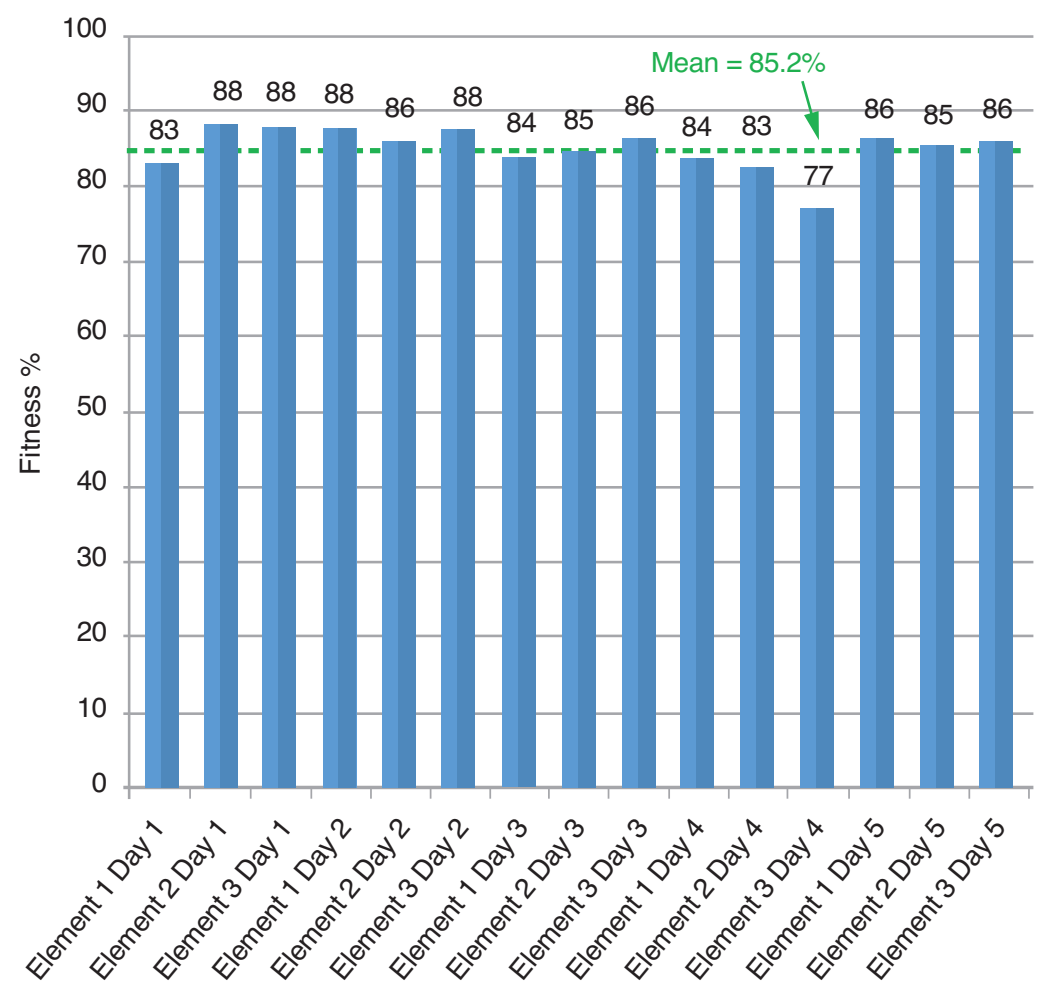

Figure 16. Fitness values of $\left[\mathrm{PPG} \rightarrow C B P_{N}\right]$ models obtained using $I T F_{\text {Ref }}$ on all 15 segments. Horizontal dotted green line: mean Fitness value.

of the predicted output $C B P_{N}$ using $I T F_{R e f}$ with the radial PPG as input are shown in Figure 16. The mean \pm 1 SD of Fitness is $85.2 \% \pm 2.5 \%$ ( $C V$ of $3.35 \%)$. The average and standard deviation differences between the model output and the maximums (peaks) of the $C B P_{N}$ signal are 0.10 and 0.03 , respectively (normalized arbitrary units). The average and standard deviation differences between the model output and the minimums (valleys) of the $C B P_{N}$ signal are 0.08 and 0.03 , respectively.

\section{DISCUSSION}

\subsection{Main Contributions}

Reliable estimation of the central blood pressure (CBP) has proven to be a key element in the diagnosis of cardiovascular diseases (CVD) [2]. Unfortunately, limitations in budget and skilled man-power often impede access to this vital information. However, valuable vascular indices can still be determined from the shape of the $\mathrm{CBP}$ waveform without knowledge of the absolute amplitude.

The proposed approach in this work can be considered a positive step towards providing the physician with relevant clinical indices, allowing for a more confident 
diagnosis. Compared to an applanation tonometer, the proposed technique is low-cost as it requires only a reflective photoplethysmography probe. Not requiring a skilled operator, it is also relatively easy to operate. Once the proper location with maximum PPG amplitude is identified, the probe remains secured in position via its wrist strap. Given the high degree of similarity of the shape of the reconstructed waveform to the estimated CBP by the de-facto standard device (SphygmoCor), one possible application could be to determine vascular indices [2] which do not require the absolute values of the waveform. A comparison of these indices using these two signals (CBP from SphygmoCor and $C B P_{N}$ as estimated via radial PPG) constitutes one of the future directions of our work.

\subsection{Study Limitations}

In the present paper we have only considered one subject, thus an important question to be addressed is the degree of similarity of the ARX models for different subjects. Our literature review shows the possibility of using either generalized (GTF) $[17,18]$ or individualized (ITF) [19] transfer functions to assess $C B P_{N}$ based on the non-invasive radial pressure. Using a GTF, there will be no need for individual calibration. However, these two techniques have been recently compared by Hahn et al. [20] showing that the ITF yields more accurate results. Regarding the sources of variation in the model's parameters, these can be categorized into three main groups: the inherent variations during numerical estimation of the model parameters due to the presence of measurement noise, the effects of the probe position/contact pressure on the PPG waveform and physiological factors. The effect of measurement noise on the ARX modeling has been mitigated by carefully limiting the frequency bandwidth to $15 \mathrm{~Hz}$ and keeping the data acquisition conditions relatively stable (subject at rest, fasting and in a controlled environment). It was demonstrated that compliance with these conditions produced satisfactory results (mean Fitness of $85.2 \%$ ).

In order to reduce the effect of the position of PPG probe over the respective radial arteries, an automatic system should be designed allowing for the determination and selection of the optimum probe position by switching an array of LED photodetectors. It is also well known that the probe contact pressure plays an important role in the genesis of the PPG [21]. At zero transmural pressure, the radial forces are eliminated and compliance is maximum (Figure 2), producing maximum amplitude PPG. In practice, this position is found with trial and error. Our experience is that if the operator is patient enough, the position of the probes (either tonometer or PPG) can be close to the optimum location. This is a routine practice with the clinical standard SphygmoCor System [22]. However, there is room for improvement as the time spent to find this optimum position is not negligible. Another factor affecting the quality of the signal is the battery voltage supplied to the PPG sensor. In our experience, the amplitude of the recorded PPG decreases whenever the battery approaches depletion. Since it is difficult to use a DC power supply due to extra power-line noise, the battery voltage must be frequently checked by the operator to ensure that the nominal voltage is supplied throughout the entire recording session.

Finally, physiological factors need to be considered in a realistic clinical setting where subjects would be selected from healthy as well as populations with risk 
factor(s). An interesting question would be to evaluate the effect of health conditions known to affect the vascular system on the models. To this end, it is necessary to extend this study with a pool of subjects chosen, for example, from the healthy, hypertensive, and diabetic subjects. Furthermore, the effects of age and gender on model variability need to be investigated.

\subsection{Future Directions}

In order to better evaluate the real potential of this method in clinical applications, the subject sample size can be increased. We are currently focusing on developing this model with more candidates among different age groups, gender and health conditions. Our primary results prove the possibility of introducing besides an individualized transfer function (ITF), and a generalized one (GTF). The question to be addressed is the amount of loss of accuracy when switching from an ITF to a GTF.

In order to better differentiate between various vascular conditions, it is also possible to elucidate a response from the radial artery by using a non-invasive stimulus such as temporary occlusion of the artery (such as flow-mediated dilation-FMD [23, 24]. This stimulus can be used to create a characteristic response from the endothelium layer, and it is logical to expect that this response will affect the transfer function (TF) between $C B P_{\mathrm{N}}$ and PPG. An interesting area would be to investigate the possibility of evaluating the endothelial function using the changes of the parameters of this TF caused by FMD.

Millasseau et al. [10] proposed a similar approach for modeling the relationship between finger PPG and BP. However, the tip of the finger may inherently be affected by the natural auto-regulation mechanism. An interesting topic would be to compare the models obtained by the two methods with the objective of inferring a more exact autoregulation mechanism in the finger. Indeed, the finger contains a microvascular bed, and it is well known that blood circulation in arterioles is prone to the effects of autoregulation [25]. The role of this auto-regulation control loop is to maintain the perfusion of the tissues independent from the blood pressure. Therefore, any time-invariant dynamic model (or transfer function if the system is considered linear) aiming at establishing a relationship between the PPG and $B P(t)$ at an anatomical location which is under the effect of auto-regulation can only remain accurate for a short period of time. As the output becomes quasi-independent from the input, it is clear that auto-regulation effects on the finger blood micro-circulation are difficult to account for by the TF, contributing to errors in the estimated $B P(t)$. The above statement may also explain why the recommended position for the tonometer is a conduit vascular structure such as the radial artery for a faithful measurement of $B P(t)$. Moreover, higher order of ARX models may provide better results in some of the subjects; therefore, this fact should be investigated to find the optimum order reflecting best model fitness for all subjects. Exploiting these features could minimize the FPE and consequently enhance the overall model fitness for a sizeable population of subjects.

Finally, it is interesting to note the difference in the frequency bands reported by various researchers. Whereas our results point to a resonance around $4 \mathrm{~Hz}$ (Figure 11 and Figure 12), Segers et al. [26] reported a frequency response with a low-impedance phenomena occurring at around $5 \mathrm{~Hz}$. Millasseau [10] reported a higher resonance at 
above $6 \mathrm{~Hz}$. Despite acknowledging the fact that the modeling and experimental conditions were different in these studies, proper interpretation of these differences will certainly shine more light on vascular dynamics.

\section{CONCLUSION}

Reliable estimation of the amplitude-normalized waveform of the central blood pressure by radial PPG has been proven to be possible using an autoregressive with exogenous input (ARX) model. It was shown that the parameters of the obtained model over five different days remain stable and the mean value of the Fitness was greater than $85 \%$ for the same subject. This technique is considerably more cost effective compared to the tonometer-based systems, and may be rendered independent from the operator's skills level in the near future with an array sensor. Our group will focus on studying the effects of age, gender as well as health conditions and the possibility of defining a single model for different subjects. Clearly, as an immediate future step, it is also possible to convert, through an appropriate calibration equation, the available estimated signal into $\mathrm{mmHg}$ dimension, to improve the practical clinical application and value of the proposed system.

\section{ACKNOWLEDGMENT}

This research was supported in part by the Ministry of Science, Technology, and Innovation (MOSTI) of Malaysia under Science Fund Grant 03-01-02-SF0703 and INDUSTRI-2013-006, PRGS/1/13/TK02/UKM/02/1.

\section{CONFLICT OF INTEREST}

The authors declare that they have no conflict of interest.

\section{NOMENCLATURE}

$A C$

ARMAX

$A R X$

$B P$

$C B P$

$C B P_{\mathrm{N}}$

$C V$

$C V D$

$D B P$

$D C$

$E C G$

EFTE

FPE

FIR

$F M D$

GTF

ITF
Alternating Current

Autoregressive moving-average with exogenous input

Autoregressive with exogenous input

Blood Pressure

Central Blood Pressure

Normalized Central Blood Pressure

Coefficient of Variation

Cardiovascular Disease

Diastolic Blood Pressure

Direct Current

Electrocardiogram

Empirical Fourier Transform Estimate

Final Prediction Error

Finite Impulse Response

Flow Mediated Dilation

General Transfer Function

Individualized Transfer Function 


$\begin{array}{ll}L P F & \text { Low Pass Filter } \\ M A P & \text { Mean Arterial Pressure } \\ N I & \text { National Instruments } \\ P_{\text {art }} & \text { Arterial Pressure } \\ P_{\text {ext }} & \text { External Pressure } \\ P P G & \text { Photoplethysmogrm } \\ P_{\mathrm{tr}} & \text { Transmural Pressure } \\ P W A & \text { Pulse Wave Analysis } \\ S B P & \text { Systolic Blood Pressure } \\ S D & \text { Standard Deviation } \\ T F & \text { Transfer Function } \\ T F_{\text {Ref }} & \text { Reference Transfer Function } \\ U K M & \text { Universiti Kebangsaan Malaysia } \\ U S & \text { Ultrasound }\end{array}$

\section{REFERENCES}

[1] M. Pavel, H. B. Jimison, H. D. Wactlar, T. L. Hayes, W. Barkis, J. Skapik, et al., "The Role of Technology and Engineering Models in Transforming Healthcare," Biomedical Engineering, IEEE Reviews in, vol. 6, pp. 156-177, 2013.

[2] A. P. Avolio, M. Butlin, and A. Walsh, "Arterial blood pressure measurement and pulse wave analysis - their role in enhancing cardiovascular assessment," Physiological measurement, vol. 31, p. $\mathrm{R} 1,2010$.

[3] C. Vlachopoulos, K. Aznaouridis, M. F. O'Rourke, M. E. Safar, K. Baou, and C. Stefanadis, "Prediction of cardiovascular events and all-cause mortality with central haemodynamics: a systematic review and meta-analysis," European heart journal, vol. 31, pp. 1865-1871, 2010.

[4] R. W. Troughton, J. Ritzema, N. L. Eigler, I. C. Melton, H. Krum, P. B. Adamson, et al., "Direct left atrial pressure monitoring in severe heart failure: long-term sensor performance," Journal of cardiovascular translational research, vol. 4, pp. 3-13, 2011.

[5] B. Fetics, E. Nevo, C. Chen, and D. A. Kass, "Parametric model derivation of transfer function for noninvasive estimation of aortic pressure by radial tonometry," Biomedical Engineering, IEEE Transactions on, vol. 46, pp. 698-706, 1999.

[6] J. Penaz, A. Voigt, and W. Teichmann, "Contribution to the continuous indirect blood pressure measurement," Zeitschrift fur die gesamte innere Medizin und ihre Grenzgebiete, vol. 31, pp. 1030-1033, 1976.

[7] Available: www.atcormedical.com/sphygmocor.html, Accessed July 2014

[8] V. Sohani, E. Zahedi, K. Chellappan, and M. A. M. Ali, "A review of commercially available noninvasive vascular screening technologies for clinical applications," in Biomedical Engineering and Sciences (IECBES), 2012 IEEE EMBS Conference on, 2012, pp. 568-573.

[9] J. G. Webster, Design of pulse oximeters: CRC Press, 2002.

[10] S. C. Millasseau, F. G. Guigui, R. P. Kelly, K. Prasad, J. R. Cockcroft, J. M. Ritter, et al., "Noninvasive assessment of the digital volume pulse comparison with the peripheral pressure pulse," Hypertension, vol. 36, pp. 952-956, 2000.

[11] L. Ljung, System Identification: theory for the user. Upper Saddle River, New Jersey 07458: Prentice Hall,, 1999.

[12] L. Wang, E. Pickwell-MacPherson, and Y. Zhang, "Blood Pressure Contour Analysis after Exercise by the Photoplethysmogram Using a Transfer Function Method," Medical Devices and Biosensors, vol. ISSS-MDBS, pp. 82-85, 2008. 
[13] P. Segers, Vascular biomechanics and hemodynamics, 2010.

[14] J. Talts, R. Raamat, and K. Jagomägi, "Asymmetric time-dependent model for the dynamic finger arterial pressure-volume relationship," Medical and Biological Engineering and Computing, vol. 44, pp. 829-834, 2006.

[15] J. Sidhu, V. Newey, D. Nassiri, and J. Kaski, "A rapid and reproducible on line automated technique to determine endothelial function," Heart, vol. 88, pp. 289-292, 2002.

[16] Available: http://www.wma.net/en/30publications/10policies/b3/. Accessed December 2014.

[17] J. E. Sharman, R. Lim, A. M. Qasem, J. S. Coombes, M. I. Burgess, J. Franco, et al., "Validation of a generalized transfer function to noninvasively derive central blood pressure during exercise," Hypertension, vol. 47, pp. 1203-1208, 2006.

[18] M. F. O'Rourke and A. Adji, "Noninvasive Generation of Aortic Pressure From Radial Pressure Waveform By Applanation Tonometry, Brachial Cuff Calibration, and Generalized Transfer Function," American journal of hypertension, vol. 27, pp. 143-145, 2014.

[19] J. O. Hahn, A. T. Reisner, F. A. Jaffer, and H. H. Asada, "Subject-specific estimation of central aortic blood pressure using an individualized transfer function: A preliminary feasibility study," Information Technology in Biomedicine, IEEE Transactions on, vol. 16, pp. 212-220, 2012.

[20] J.-O. Hahn, "Individualized Estimation of the Central Aortic Blood Pressure Waveform: A Comparative Study," Biomedical and Health Informatics, IEEE Journal of, vol. 18, pp. 215-221, 2014.

[21] A. Grabovskis, Z. Marcinkevics, U. Rubins, and E. Kviesis-Kipge, "Effect of probe contact pressure on the photoplethysmographic assessment of conduit artery stiffness," Journal of biomedical optics, vol. 18, pp. 027004-027004, 2013.

[22] C. Geoffrey, C. Rajkumar, J. Kooner, J. Cooke, and C. J. Bulpitt, "Estimation of central aortic pressure by SphygmoCor ${ }^{\circledR}$ requires intra-arterial peripheral pressures," Clinical Science, vol. 105, pp. 219-225, 2003.

[23] E. Zahedi, R. Jaafar, M. M. Ali, A. Mohamed, and O. Maskon, "Finger photoplethysmogram pulse amplitude changes induced by flow-mediated dilation," Physiological measurement, vol. 29, p. 625, 2008.

[24] M. Zaheditochai, R. Jaafar, and E. Zahedi, "Non-invasive techniques for assessing the endothelial dysfunction: ultrasound versus photoplethysmography," in 13th International Conference on Biomedical Engineering, 2009, pp. 65-68.

[25] P. A. S. A. Reisner, D. McCombie and H. Asada, "Utility of the photoplethysmogram in circulatory monitoring," Anesthesiology, vol. 108, pp. 950-958, 2008.

[26] P. Segers, N. Stergiopulos, P. Verdonck, and R. Verhoeven, "Assessment of distributed arterial network models," Medical and Biological Engineering and Computing, vol. 35, pp. 729-736, 1997.

\section{APPENDIX A. LOW-PASS FILTER CODE IN MATLAB}

$\mathrm{F}_{\mathrm{S}}=1613$

$\mathrm{F}_{\text {pass }}=10$

$\mathrm{F}_{\text {stop }}=40$;

$\mathrm{D}_{\text {pass }}=0.057501127785$

$\mathrm{D}_{\text {stop }}=0.0001$;

dens $=20$;
$\%$ Sampling Frequency

$\%$ Passband Frequency

$\%$ Stopband Frequency

$\%$ Passband Ripple

$\%$ Stopband Attenuation

$\%$ Density Factor

$\%$ Calculate the order from the parameters using FIRPMORD.

$[\mathrm{N}, \mathrm{Fo}, \mathrm{Ao}, \mathrm{W}]=$ firpmord $\left(\left[\mathrm{F}_{\text {pass }}, \mathrm{F}_{\text {stop }}\right] /\left(\mathrm{F}_{\mathrm{s}} / 2\right),[10],\left[\mathrm{D}_{\text {pass }}, \mathrm{D}_{\text {stop }}\right]\right)$;

$\%$ Calculate the coefficients using the FIRPM function.

$\mathrm{b}=\operatorname{firpm}(\mathrm{N}$, Fo, Ao, $\mathrm{W},\{$ dens $\})$;

$\mathrm{Hd}=$ dfilt.dffir(b); 
APPENDIX B. COMPUTATION OF THE CONFIDENCE INTERVAL

Given the general weighted least square error $\hat{\theta}_{N}$ and the covariance matrix of $\hat{\theta}_{N}=p_{N}$, the confidence interval is defined as [11]:

$$
\hat{\theta}_{N}-\theta_{0} \in N\left(0, p_{N}\right)
$$

where $\mathrm{N}\left(0, p_{N}\right)$ is the zero mean normal distribution with variance $p_{N}$. For the $\mathrm{i}^{\text {th }}$ component of $\hat{\theta}_{N}$,

$$
\frac{\hat{\theta}_{N}^{i}-\theta_{0}^{i}}{\sqrt{p_{N}^{i i}}} \in N(0,1)
$$

where $p_{N}^{i i}$ indicates the $\mathrm{i}^{\text {th }}$ diagonal element of $\mathrm{P}_{\mathrm{N}}$. In fact the last equation defines an ellipsoidal shape with a width determined by $p_{N}$. 


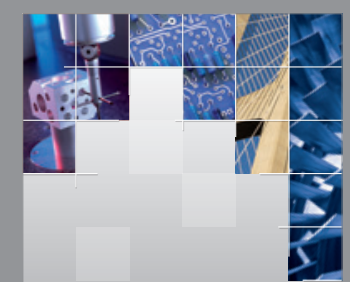

\section{Enfincering}
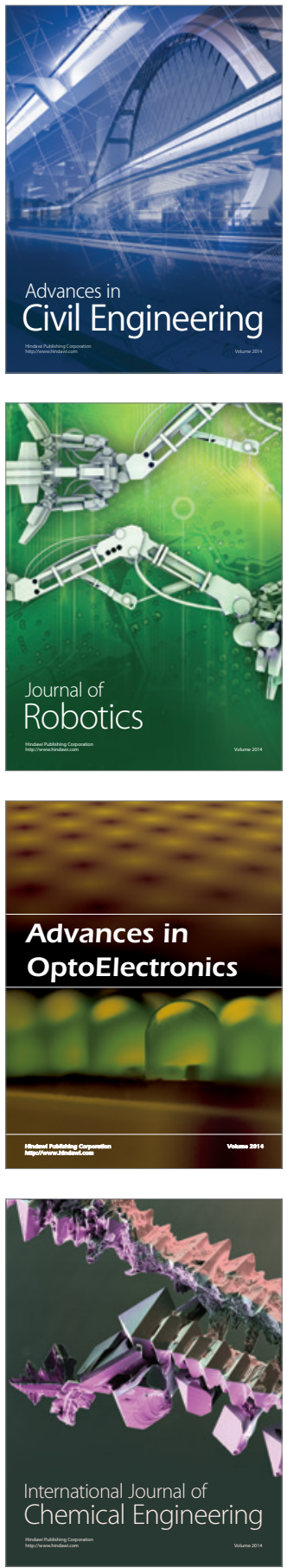

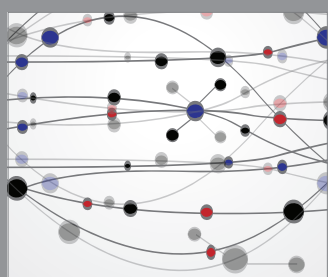

The Scientific World Journal

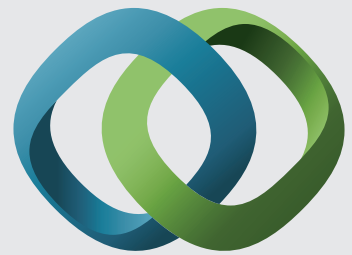

\section{Hindawi}

Submit your manuscripts at

http://www.hindawi.com
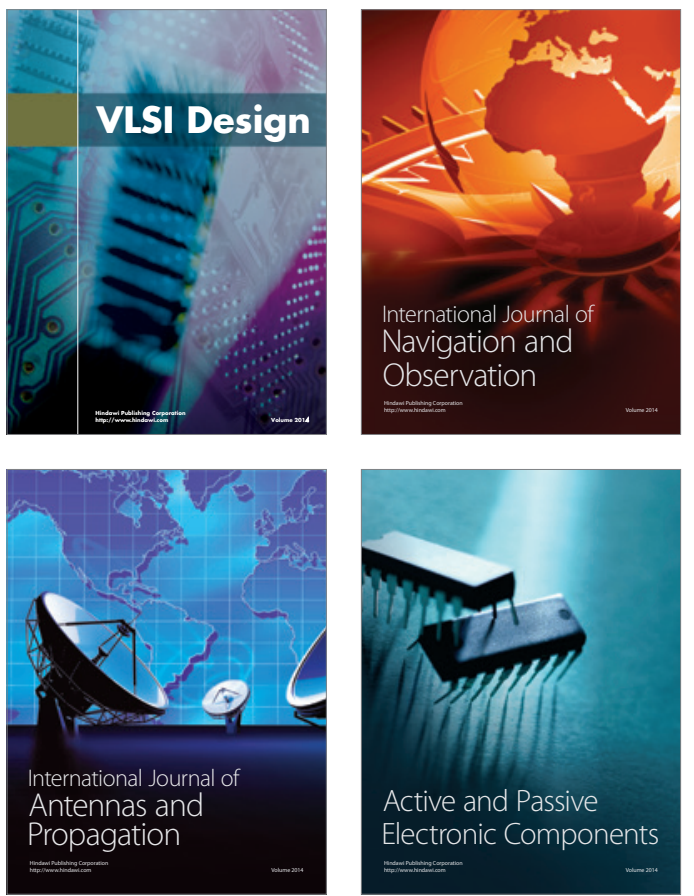
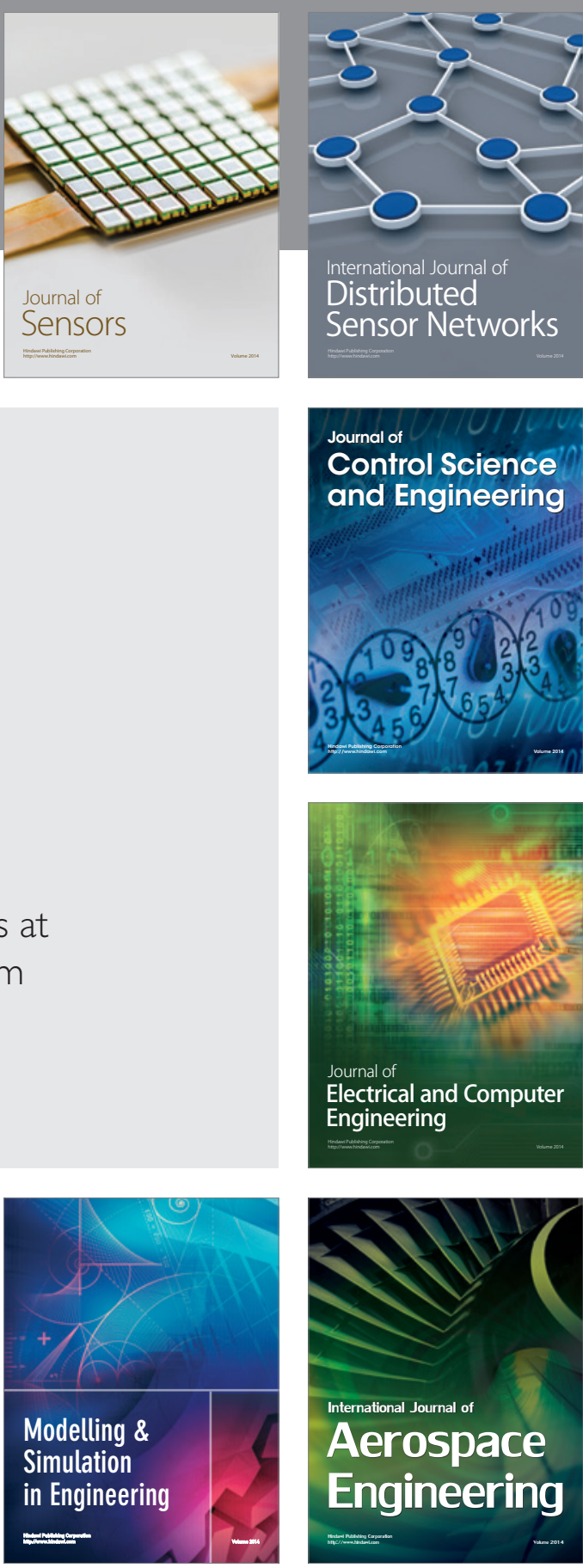

International Journal of

Distributed

Sensor Networks

Journal of

Control Science

and Engineering
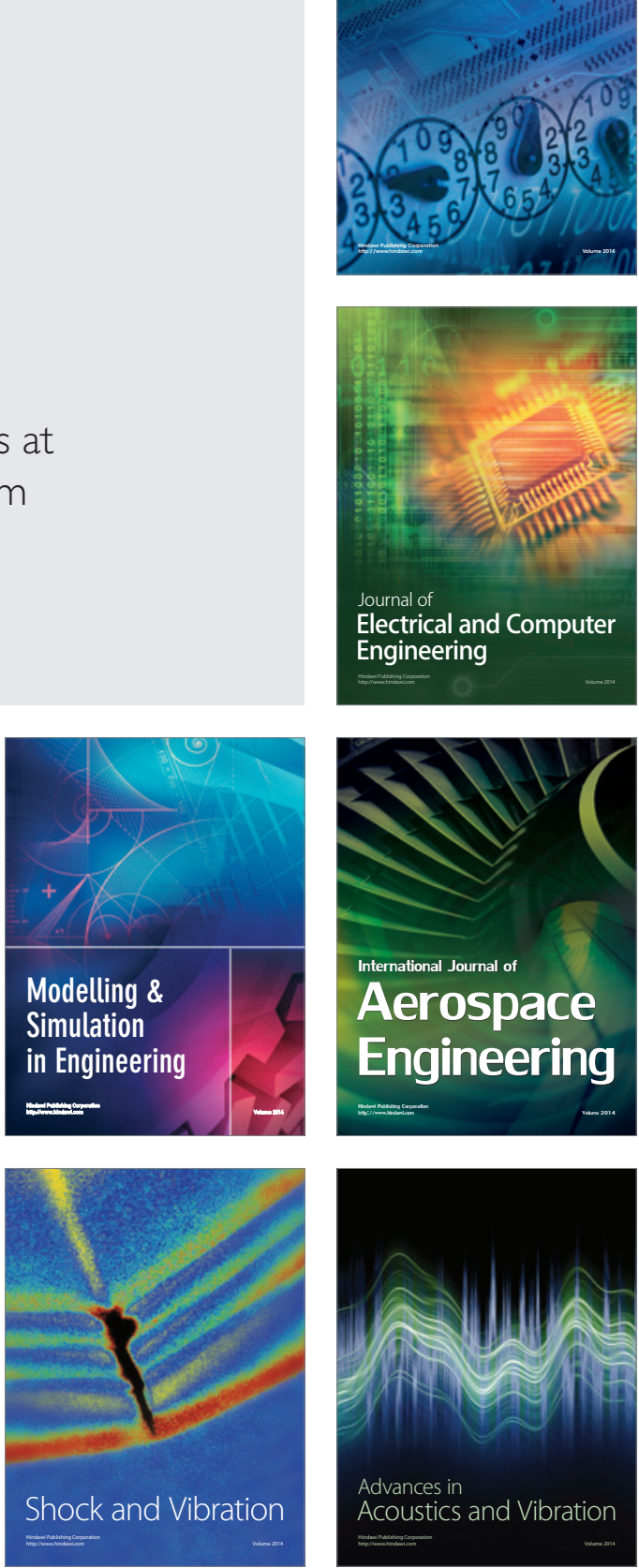